\title{
QUASISYMMETRIC DISTORTION AND RIGIDITY OF EXPANDING ENDOMORPHISMS OF $S^{1}$
}

\author{
EDSON DE FARIA
}

(Communicated by Linda Keen)

\begin{abstract}
In this paper we examine a result of D. Sullivan according to which two $C^{1+\alpha}$ expanding endomorphisms of the circle are $C^{1+\alpha}$ conjugate as soon as they are symmetrically conjugate. We develop general a priori estimates on the local distortion of quasisymmetric mappings and combine them with the classical naive distortion lemma to present a complete proof of Sullivan's result. A new proof is offered at the end that renders unnecessary the use of Markov partitions or the control of eigenvalues at periodic points.
\end{abstract}

\section{INTRODUCTION}

We say that a $C^{1}$ endomorphism $F: S^{1} \rightarrow S^{1}$ is expanding if $|D F(z)|>1$ for all $z \in S^{1}$. Such an $F$ is easily seen to be a covering map, and if we let $d$ be its topological degree $(|d| \geq 2$, necessarily), a well-known theorem of M. Shub [Sh] states that $F$ is topologically conjugate to $z \mapsto z^{d}$. The conjugacy is in general not smooth, and it may even be purely singular with respect to Lebesgue measure.

One way to remedy this situation is to impose further smoothness on $F$. Thus, if $F$ is $C^{1+\alpha}$, i.e. if $D F$ is $\alpha$-Hölder continuous, one can show that, although still arbitrarily bad from the differentiable viewpoint, the conjugacy in question is quasisymmetric (cf. [Su1]). Recall that a mapping is quasisymmetric if it distorts symmetric triples of points in its domain by only a bounded amount. If the distortion goes to zero uniformly with the size of the triples, we say that the mapping is symmetric, and if this last property holds only locally at some point $q$, we say that the mapping is symmetric at $q$ (see $\S 2$ ). In this note, our aim is to develop certain a priori distortion estimates for quasisymmetric as well as symmetric mappings, in order to give a complete proof of the following rigidity theorem, first stated by D. Sullivan in [Su2] in a slightly weaker form.

Theorem. If the conjugacy between two $C^{1+\alpha}$ expanding endomorphisms of $S^{1}$ is symmetric at a point, then it is $C^{1+\alpha}$.

The main tool to be employed here is the following classical result, the proof of which is a standard geometric series majorization argument (cf. [SS]). If $\varphi$ is any $\alpha$-Hölder continuous function on an interval in $\mathbf{R}$ or $S^{1}$, we let $\|\varphi\|_{\alpha}$ denote its $\alpha$-Hölder norm there.

Received by the editors November 21, 1994.

1991 Mathematics Subject Classification. Primary 58F03, 30C62; Secondary 26A16.

Key words and phrases. Expanding maps, symmetric conjugacies.

This work is part of Projeto Temático de Equipe "Transição de Fase Dinâmica em Sistemas Evolutivos", supported by FAPESP Grant 90/3918-5. 
Distortion Lemma. Let $\left\{f_{n}: I_{n} \rightarrow I_{n+1}\right\}_{n \geq 1}$ be a sequence of $C^{1+\alpha}$ diffeomorphisms between intervals $I_{n}$ in $\mathbf{R}$ or $S^{1}$, and assume that $\left|D f_{n}(x)\right| \leq \lambda<1$ for all $x \in I_{n}$ and all $n \geq 1$. If $\beta=\lambda^{\alpha} \sup \left\|\log \left|D f_{n}\right|\right\|_{\alpha}^{1 / n}<1$, then for all $x, y \in I_{1}$ and all $n \geq 1$

$$
|\log | \frac{D\left(f_{n} \circ f_{n-1} \circ \cdots \circ f_{1}\right)(x)}{D\left(f_{n} \circ f_{n-1} \circ \cdots \circ f_{1}\right)(y)}|| \leq\left(\frac{\lambda^{-\alpha} \beta}{1-\beta}\right)|x-y|^{\alpha} .
$$

The hypotheses in this lemma are obviously satisfied if we let $F$ be a $C^{1+\alpha}$ expanding endomorphism of the circle and accordingly take each $f_{n}$ to be an appropriate branch of $F^{-1}$, and it is only in this context that the lemma will be used.

\section{Quasisymmetric Distortion}

The length of an interval $I$ in $\mathbf{R}$ or $S^{1}$ is denoted by $|I|$; if $x, y \in I$, the distance between $x$ and $y$ measured along $I$ is denoted by either $d_{I}(x, y)$ or $d(x, y ; I)$.

Let $I_{0}, I_{1}$ be closed intervals in $\mathbf{R}$ or $S^{1}$, and let $\psi: I_{0} \rightarrow I_{1}$ be an orientationpreserving homeomorphism. If $T \subseteq I_{0}$ is a sub-interval we define the quasisymmetric distortion $M_{T}=M(\psi, T)$ of the restriction $\psi \mid T$ to be the smallest $C>1$ satisfying $C^{-1}|\psi(J)| \leq|\psi(I)| \leq C|\psi(J)|$ for all pairs of adjacent intervals $I, J \subseteq T$ of equal length. The map $\psi$ is called (M-)quasisymmetric if $M_{T} \leq M<\infty$ for all $T \subseteq I_{0}$. If $M_{T} \rightarrow 1$ uniformly in $T$ as $|T| \rightarrow 0$ for intervals $T$ containing a given point $q$, then we say that $\psi$ is symmetric at $q$. A homeomorphism which is symmetric at all points of its domain is called symmetric. Symmetric homeomorphisms were first investigated in [GS1].

We shall need three lemmas on quasisymmetry. They are standard results in the theory of quasiconformal mappings. For instance, Lemma 1 simply says that quasisymmetric mappings are Hölder continuous. The classical proofs of these results, as given say in $[\mathrm{Ah}]$, are based on the fact that quasisymmetric mappings are precisely the boundary values of quasiconformal mappings (in the upper half-plane or unit disk), and on certain inequalities involving the dilatations of qc-mappings. For convenience, we present intrinsic one-dimensional proofs of these lemmas.

Lemma 1. Let $\psi: I_{0} \rightarrow I_{1}$ be a quasisymmetric homeomorphism. Then, if $I \subseteq T$ are intervals in $I_{0}$ sharing an endpoint and satisfying $\theta=|I| /|T| \leq 1 / 2$, we have

$$
\theta^{\beta_{T}(\theta)} \leq \frac{|\psi(I)|}{|\psi(T)|} \leq \theta^{\gamma_{T}(\theta)}
$$

where $\beta_{T}(\theta)=\left(1+\frac{1}{m-1}\right) \log _{2}\left(1+M_{T}\right), \gamma_{T}(\theta)=\left(1-\frac{1}{m}\right) \log _{2}\left(1+M_{T}^{-1}\right)$ and $m \geq 2$ is the unique integer such that $2^{-m}<\theta \leq 2^{-(m-1)}$.

Proof. For each $n \geq 0$ let $T_{n} \subseteq T$ be the unique closed sub-interval of length $2^{-n}|T|$ sharing an endpoint with both $T$ and $I$. Since $\psi \mid T$ is $M_{T}$-quasisymmetric, we have

$$
\frac{1}{1+M_{T}} \leq \frac{\left|\psi\left(T_{n+1}\right)\right|}{\left|\psi\left(T_{n}\right)\right|} \leq \frac{M_{T}}{1+M_{T}}
$$


Taking into account the decomposition $\left|\psi\left(T_{n}\right)\right| /|\psi(T)|=\prod_{i=0}^{n-1}\left(\left|\psi\left(T_{i+1}\right)\right| /\left|\psi\left(T_{i}\right)\right|\right)$, we deduce that

$$
\left(\frac{\left|T_{n}\right|}{|T|}\right)^{\log _{2}\left(1+M_{T}\right)} \leq \frac{\left|\psi\left(T_{n}\right)\right|}{|\psi(T)|} \leq\left(\frac{\left|T_{n}\right|}{|T|}\right)^{\log _{2}\left(1+M_{T}^{-1}\right)},
$$

for all $n \geq 0$.

Now, if $m=\left[\log \theta^{-1}\right]+1$, we have $T_{m} \subseteq I \subseteq T_{m-1}$. Therefore, using the last inequality in (1) we get

$$
\frac{|\psi(I)|}{|\psi(T)|} \leq\left(\frac{\left|T_{m-1}\right|}{|T|}\right)^{\log _{2}\left(1+M_{T}^{-1}\right)}=\left(\frac{\left|T_{m}\right|}{|T|}\right)^{\gamma_{T}(\theta)} \leq \theta^{\gamma_{T}(\theta)}
$$

which proves one half of Lemma 1 . The other half follows similarly from the first inequality in (1).

Lemma 2. Given a quasisymmetric homeomorphism $\psi$ and an interval $T$ in its domain, let $A$ be the unique affine, orientation-preserving homeomorphism that maps $T$ onto $\psi(T)$. Then $d_{\psi(T)}(\psi(x), A(x)) \leq \frac{1}{2}\left(M_{T}-1\right)|\psi(T)|$ for all $x \in T$.

Proof. We may assume that $T=\psi(T)=[0,1]$, so that $A(x)=x$ for all $x$. The general case follows from this one by affine changes of coordinates. We let $M$ be $M_{[0,1]}$ in this proof.

Let $\left(\psi_{n}\right)_{n \geq 0}$ be the successive dyadic approximations of $\psi \mid[0,1]$. This means that $\psi_{n}:[0,1] \rightarrow[0,1]$ agrees with $\psi$ at the points $\left\{j 2^{-n}: j=0,1, \cdots, 2^{n}\right\}$ determining the $n$-th dyadic partition of $[0,1]$ and is affine on each interval of that partition. Clearly, $\left(\psi_{n}\right) \rightarrow \psi$ in the $C^{0}$ topology. Writing $\mu=M /(1+M)$, an easy inductive argument using the quasisymmetric condition satisfied by $\psi$ shows us that

$$
\psi\left(\frac{j+1}{2^{n}}\right)-\psi\left(\frac{j}{2^{n}}\right) \leq \mu^{n}
$$

for all $j, 0 \leq j<2^{n}$, and all $n \geq 0$.

Now let $d_{n}=\sup _{t \in[0,1]}\left|\psi_{n}(t)-t\right|$. Then we have $d_{0}=0$ and $d_{1} \leq \mu-\frac{1}{2}=$ $\frac{1}{2}\left(\frac{M-1}{M+1}\right)$, while from (2) we get $d_{n} \leq d_{n-1}+d_{1} \mu^{n-1}$, which gives us

$$
d_{n} \leq d_{1}(1-\mu)^{-1} \leq \frac{1}{2}(M-1) .
$$

Letting $n \rightarrow \infty$ we deduce that $\sup _{t \in[0,1]}|\psi(t)-t| \leq \frac{1}{2}(M-1)$, as was to be proved.

Remark. Obviously, the estimate provided by Lemma 2 yields non-trivial information only when $M_{T}$ is near 1 , as it happens at small scales when $\psi$ is symmetric. This estimate is related to Ahlfors' maximal function (cf. [Ah, page 67]).

Our third lemma states that the composition of two quasisymmetric mappings with small local distortion still has small local distortion. It is by no means the sharpest result of its kind. 
Lemma 3. Given $0 \leq \varepsilon \leq 1 / 4$ and intervals $I_{i}, i=0,1,2$, let $\psi_{0}: I_{0} \rightarrow I_{1}$ and $\psi_{1}: I_{1} \rightarrow I_{2}$ be quasisymmetric homeomorphisms such that $M\left(\psi_{0}, I_{0}\right) \leq 1+\varepsilon$ and $M\left(\psi_{1}, I_{1}\right) \leq 1+\varepsilon$ respectively. Then $M\left(\psi_{1} \circ \psi_{0}, I_{0}\right) \leq 1+3 \sqrt{\varepsilon}$.

Proof. Let us write $M\left(\psi_{i}, I_{i}\right)=1+\varepsilon_{i}$, so that $\varepsilon_{i} \leq \varepsilon$. Taking $J$ and $J^{*}$ to be any two intervals in $I_{0}$ of the same length and adjacent at some point $p$, we let $T=\psi_{0}\left(J^{*}\right)$. We also let $\Delta$ be the interval symmetric to $\psi_{0}(J)$ about $\psi_{0}(p)$ inside $I_{1}$ and consider the interval $I=T \backslash \Delta$. Here we assume that $\Delta \subseteq T$; otherwise we simply interchange $J$ and $J^{*}$. Then we have

$$
\frac{\left|\psi_{1} \psi_{0}(J)\right|}{\left|\psi_{1} \psi_{0}\left(J^{*}\right)\right|}=\frac{\left|\psi_{1}\left(\psi_{0} J\right)\right|}{\left|\psi_{1}(\Delta)\right|} \frac{\left|\psi_{1}(\Delta)\right|}{\left|\psi_{1}(T)\right|} .
$$

This expression is clearly bounded above by $1+\varepsilon_{1}$, so we only need a good lower bound. The first ratio in the second member of (3) is bounded below by $\left(1+\varepsilon_{1}\right)^{-1}$. From the fact that $\psi_{0}$ is $\left(1+\varepsilon_{0}\right)$-quasisymmetric, we also see that $\theta=|I| /|T| \leq$ $\varepsilon_{0} /\left(1+\varepsilon_{0}\right)$. Therefore Lemma 1 applied to $\psi=\psi_{1}$ yields

$$
\frac{\left|\psi_{1}(\Delta)\right|}{\left|\psi_{1}(T)\right|} \geq 1-\theta^{\gamma_{T}(\theta)} .
$$

Since here, in the notation of Lemma 1 , we have $m=m(\theta) \geq 3$ as well as $M_{T} \leq$ $1+\varepsilon_{0} \leq 5 / 4$, it follows that

$$
\gamma_{T}(\theta) \geq \frac{2}{3} \log _{2} \frac{9}{5}>\frac{1}{2} .
$$

Carrying these facts back into (3), we get

$$
\frac{\left|\psi_{1} \psi_{0}(J)\right|}{\left|\psi_{1} \psi_{1}\left(J^{*}\right)\right|} \geq \frac{1-\sqrt{\varepsilon_{0}}}{1+\varepsilon_{1}} \geq \frac{1-\sqrt{\varepsilon}}{1+\varepsilon},
$$

which finally gives us

$$
M\left(\psi_{1} \psi_{0}, I_{0}\right) \leq \frac{1+\varepsilon}{1-\sqrt{\varepsilon}} \leq 1+3 \sqrt{\varepsilon} .
$$

\section{Proof of the THEOREM}

Let $F$ be a $C^{1}$ endomorphism of the circle and $p \in S^{1}$ be $F$-periodic of minimum period $k>0$. Then the eigenvalue of $p$ is defined as $\lambda_{F}(p)=\left|\left(F^{k}\right)^{\prime}(p)\right|$. We start by examining the relationship between quasisymmetric distortion of a conjugacy and eigenvalues at corresponding periodic points.

Proposition 4. Let $F$ and $G$ be $C^{1+\alpha}$ expanding endomorphisms of the circle, and let $h: S^{1} \rightarrow S^{1}$ be a quasisymmetric conjugacy between them. If $p \in S^{1}$ is a periodic point of $F$ and $T$ is any interval in $S^{1}$ containing $p$, then

$$
\left|1-\frac{\log \lambda_{G}(h(p))}{\log \lambda_{F}(p)}\right| \leq \log _{2} M_{T} .
$$


Proof. To simplify the notation, we shall write $\lambda_{F}, \lambda_{G}$ instead of $\lambda_{F}(p), \lambda_{G}(h(p))$ in this proof. There is no loss of generality in assuming that $p$ is an endpoint of $T$. Let $k>0$ be the smallest period of $p$, and for each $n \geq 0$ let $I_{n}$ be the image of $T$ under the inverse branch of $F^{n k}$ that fixes $p$. For the purpose of what follows, we may assume also that $F$ is orientation preserving. In this case, since $F$ is expanding, we have both $I_{n} \subseteq T$ and $h\left(I_{n}\right) \subseteq h(T)$ for all $n \geq 0$.

By the Distortion Lemma and the mean value theorem applied to the composition $F^{-n k}$, we have

$$
\left|\log \frac{\left|I_{n}\right|}{|T|}-\log \left(\lambda_{F}^{-n}\right)\right| \leq c_{1}|T|^{\alpha}
$$

for all $n \geq 0$ and some positive constant $c_{1}$. The same procedure applied to $G^{-n k}$ yields

$$
\left|\log \frac{\left|h\left(I_{n}\right)\right|}{|h(T)|}-\log \left(\lambda_{G}^{-n}\right)\right| \leq c_{2}|h(T)|^{\alpha}
$$

for all $n \geq 0$ and some positive constant $c_{2}$. On the other hand, setting $\theta_{n}=\left|I_{n}\right| /|T|$ we have $\theta_{n}<1 / 2$ for every sufficiently large $n$, and therefore by Lemma 1

$$
\beta_{T}\left(\theta_{n}\right) \log \theta_{n} \leq \log \frac{\left|h\left(I_{n}\right)\right|}{|h(T)|} \leq \gamma_{T}\left(\theta_{n}\right) \log \theta_{n}
$$

Taking into account that $\log \theta_{n}<0$, this may be rewritten as

$$
\left|\log \frac{\left|h\left(I_{n}\right)\right|}{|h(T)|}-\log \frac{\left|I_{n}\right|}{|T|}\right| \leq\left(\beta_{T}\left(\theta_{n}\right)-\gamma_{T}\left(\theta_{n}\right)\right)\left|\log \theta_{n}\right| .
$$

Putting (5), (6) and (7) together, we get

$$
\left|\log \lambda_{F}-\log \lambda_{G}\right| \leq \frac{1}{n}\left\{c_{1}|T|^{\alpha}+c_{2}|h(T)|^{\alpha}+\left[\beta_{T}\left(\theta_{n}\right)-\gamma_{T}\left(\theta_{n}\right)\right]\left|\log \theta_{n}\right|\right\}
$$

for all sufficiently large $n$. Notice however that (5) also yields $\left|\log \theta_{n}\right| \leq c_{1}|T|^{\alpha}+$ $n . \log \lambda_{F}$. Furthermore

$$
\lim _{\theta \rightarrow 0}\left[\beta_{T}(\theta)-\gamma_{T}(\theta)\right]=\log _{2}\left(1+M_{T}\right)-\log _{2}\left(1+M_{T}^{-1}\right)=\log _{2} M_{T} .
$$

Carrying these facts into the above inequality and letting $n \rightarrow \infty$, we finally get

$$
\left|\log \lambda_{F}-\log \lambda_{G}\right| \leq\left(\log _{2} M_{T}\right)\left(\log \lambda_{F}\right) .
$$

Proof of Theorem. Let $F$ and $G$ be the two expanding endomorphisms in the statement, and let $h$ be the conjugacy between them. We divide the proof into four steps, gradually improving upon the smoothness of $h$. 
Step 1: Symmetry at one point implies symmetry. For $\varepsilon>0$, let $\delta_{\varepsilon}>0$ be the smallest with the property that $|h(J)| \leq \delta_{\varepsilon}$ whenever $|J| \leq \varepsilon$; observe that $\delta_{\varepsilon} \downarrow 0$ as $\varepsilon \downarrow 0$. Now let $h$ be symmetric at $q \in S^{1}$ and choose a non-degenerate interval $I$ containing $q$ such that $M(h, I) \leq 1+\varepsilon$. Let $z_{0} \in \operatorname{int}(I)$ be a point whose forward orbit $\left\{z_{n}=F^{n}\left(z_{0}\right)\right\}_{n \geq 0}$ is dense in the circle; then in fact $\left\{z_{n}\right\}_{n \geq N}$ is dense also, for all $N \geq 0$. Since $F$ is expanding, if $N$ is sufficiently large, then for all $n \geq N$ we can find an interval $I_{n} \subseteq I$ centered at $z_{0}$ such that

$$
c \varepsilon \leq\left|J_{n}\right| \leq \varepsilon,
$$

where $J_{n}=F^{n}\left(I_{n}\right)$ and $0<c<1$ is a constant depending only on $F$. Using the fact that $z_{n}$ is not far from the center of $J_{n}$ relative to its length, we see that $\left\{\operatorname{int}\left(J_{n}\right)\right\}_{n \geq N}$ covers the circle. By the Distortion Lemma, there exists $B_{F}>0$ such that

$$
M\left(F^{-n}, J_{n}\right) \leq \exp \left\{B_{F}\left|J_{n}\right|^{\alpha}\right\}<1+2 B_{F} \varepsilon^{\alpha},
$$

provided $\varepsilon$ is sufficiently small. Likewise, there exists $B_{G}>0$ such that

$$
M\left(G^{n}, h(I)\right) \leq \exp \left\{B_{G}\left|h\left(J_{n}\right)\right|^{\alpha}\right\}<1+2 B_{G} \delta_{\varepsilon}^{\alpha},
$$

for sufficiently small $\varepsilon$. Using $M(h, I) \leq 1+\varepsilon$ and (8), we get by Lemma 3

$$
M\left(h F^{-n}, J_{n}\right) \leq 1+3 \sqrt{\sigma_{\varepsilon}},
$$

where $\sigma_{\varepsilon}=\max \left\{2 B_{F} \varepsilon^{\alpha}, \varepsilon\right\}$, provided $\varepsilon$ is sufficiently small. Combining (9) and (10) and applying Lemma 3 again we obtain

$$
M\left(h, J_{n}\right)=M\left(G^{n} h F^{-n}, J_{n}\right) \leq 1+3 \sqrt{\omega_{\varepsilon}},
$$

where now $\omega_{\varepsilon}=\max \left\{2 B_{G} \delta_{\varepsilon}^{\alpha}, 3 \sqrt{\sigma_{\varepsilon}}\right\}$, for sufficiently small $\varepsilon$. Since $\omega_{\varepsilon} \downarrow 0$ as $\varepsilon \downarrow 0$, we deduce that $h$ is indeed a symmetric homeomorphism.

Step 2: Eigenvalues at corresponding periodic points are equal. This step is now a clear corollary to Proposition 4, for if $h$ is symmetric, then $M_{T} \rightarrow 1$ as $|T| \rightarrow 0$ and so the first member of (4) must vanish for every $F$-periodic point $p \in S^{1}$.

Step 3: The conjugacy is bi-Lipschitz. This in turn becomes a fairly standard Markov partition argument. Let $z \in S^{1}$ be, say, a fixed point for $F$. For each $n \geq 0$, let $\mathcal{M}_{F}^{(n)}$ be the partition of $S^{1}$ determined by the $d^{n}$ points of $F^{-n}(z)$, where $d=$ degree of $F$. Using $G^{-n}(h z)$, define $\mathcal{M}_{G}^{(n)}$ for each $n \geq 0$ in similar fashion. Notice that $h$ induces an isomorphism $\mathcal{M}_{F}^{(n)} \rightarrow \mathcal{M}_{G}^{(n)}$ for each $n \geq 0$. If $I \in \mathcal{M}_{F}^{(n)}$, then $F^{n}(I)=S^{1}$ and in fact $n$ is the smallest with this property. If $F^{-n}$ denotes the inverse branch of $F^{n}$ that maps $S^{1}-\{z\}$ onto int $(I)$, then, by the mean value theorem, $|I|=\left|\left(F^{-n}\right)^{\prime}(\xi)\right| . L$ where $L$ is the length of $S^{1}$ and $\xi \in S^{1}-\{z\}$. Similarly, $|h(I)|=\left|\left(G^{-n}\right)^{\prime}(\eta)\right| . L$, and so we have

$$
\frac{|h(I)|}{|I|}=\frac{\left|\left(G^{-n}\right)^{\prime}(\eta)\right|}{\left|\left(F^{-n}\right)^{\prime}(\xi)\right|} .
$$


But both $I$ and $h(I)$ contain points $p$ and $h(p)$ respectively such that $F^{n}(p)=p$, $G^{n}(h(p))=h(p)$. By Step 2 the corresponding eigenvalues are equal. Using the Distortion Lemma we deduce that there exists $B>1$ such that $B^{-1}|I| \leq|h(I)| \leq$ $B|I|$ for all $I \in \mathcal{M}_{F}^{(n)}$ and all $n \geq 0$.

Finally, if $T \subseteq S^{1}$ is a non-degenerate interval and $\mathcal{O}_{n}(T)=\left\{I \in \mathcal{M}_{F}^{(n)}: I \subseteq T\right\}$, then

$$
B^{-1} \sum_{I \in \mathcal{O}_{n}(T)}|I| \leq \sum_{I \in \mathcal{O}_{n}(T)}|h(I)| \leq B \sum_{I \in \mathcal{O}_{n}(T)}|I|,
$$

and if we let $n \rightarrow \infty$ we get $B^{-1}|T| \leq|h(T)| \leq B|T|$. This proves $h$ is bi-Lipschitz as asserted.

Step 4: The conjugacy is $C^{1+\alpha}$. Let $T \subseteq S^{1}$ be a closed interval and select an infinite backward branch sequence: $\cdots \stackrel{F}{\longrightarrow} T_{n} \stackrel{F}{\longrightarrow} T_{n-1} \longrightarrow \cdots \stackrel{F}{\longrightarrow} T_{0}=T$, where each arrow is a diffeomorphism. We have a corresponding sequence $\cdots \stackrel{G}{\longrightarrow}$ $h\left(T_{n}\right) \stackrel{G}{\longrightarrow} h\left(T_{n-1}\right) \longrightarrow \cdots \stackrel{G}{\longrightarrow} h\left(T_{0}\right)=h(T)$. For each $n \geq 0$, let $A_{n}: T_{n} \rightarrow h\left(T_{n}\right)$ be the unique affine orientation-preserving homeomorphism between those intervals and set $h_{n}=G^{n} \circ A_{n} \circ F^{-n}: T \rightarrow h(T)$.

We claim that $\left(h_{n}\right)_{n>0} \rightarrow h \mid T$ in the $C^{0}$ topology as $n \rightarrow \infty$. Note we can write $h=G^{n} \circ h \circ F^{-n}$ for each $n \geq 0$ because $h$ is a conjugacy. Hence, if $x \in T$ we have for all $n \geq 0$

$$
\begin{gathered}
d_{h(T)}\left(h_{n}(x), h(x)\right)=d_{h(T)}\left(G^{n} \circ A_{n} \circ F^{-n}(x), G^{n} \circ h \circ F^{-n}(x)\right) \\
=\left|\left(G^{n}\right)^{\prime}\left(\xi_{n}\right)\right| . d_{h\left(T_{n}\right)}\left(h \circ F^{-n}(x), A_{n} \circ F^{-n}(x)\right)
\end{gathered}
$$

for some $\xi_{n} \in h\left(T_{n}\right)$, by the mean value theorem. Applying Lemma 2 and using the $B$-Lipschitz condition on $h$ given by Step 3, we have

$$
d_{h\left(T_{n}\right)}\left(h \circ F^{-n}(x), A_{n} \circ F^{-n}(x)\right) \leq \frac{1}{2}\left(M_{T_{n}}-1\right) B\left|T_{n}\right| .
$$

But by the mean value theorem, $\left|T_{n}\right|=\left|\left(F^{-n}\right)^{\prime}\left(\eta_{n}\right)\right| \cdot|T|$ for some $\eta_{n} \in T$. Going back to (12) we obtain

$$
d_{h(T)}\left(h_{n}(x), h(x)\right) \leq \frac{1}{2}\left(M_{T_{n}}-1\right) B|T|\left\{\frac{\left|\left(F^{-n}\right)^{\prime}\left(\eta_{n}\right)\right|}{\left|\left(G^{-n}\right)^{\prime}\left(G^{n} \xi_{n}\right)\right|}\right\},
$$

where we have used the chain rule. We bound the term between brackets from above as follows. By the Distortion Lemma, there exists a constant $C_{1}>1$ such that for all $y \in T$ and all $n \geq 0$

$$
C_{1}^{-1} \leq \frac{\left|\left(F^{-n}\right)^{\prime}\left(\eta_{n}\right)\right|}{\left|\left(F^{-n}\right)^{\prime}(y)\right|} \leq C_{1} .
$$

Similarly, there exists $C_{2}>1$ such that for all $z \in h(T)$ and all $n \geq 0$

$$
C_{2}^{-1} \leq \frac{\left|\left(G^{-n}\right)^{\prime}(z)\right|}{\left|\left(G^{-n}\right)^{\prime}\left(G^{n} \xi_{n}\right)\right|} \leq C_{2} .
$$


Choosing $y$ and $z$ so that $\left|\left(F^{-n}\right)^{\prime}(y)\right|=\left|T_{n}\right| /|T|$ and $\left|\left(G^{-n}\right)^{\prime}(z)\right|=\left|h\left(T_{n}\right)\right| /|h(T)|$ and multiplying the last inequalities of both (14) and (15) term by term yields

$$
\frac{\left|\left(F^{-n}\right)^{\prime}\left(\eta_{n}\right)\right|}{\left|\left(G^{-n}\right)^{\prime}\left(G^{n} \xi_{n}\right)\right|} \leq C_{1} C_{2} \frac{\left|T_{n}\right|}{\left|h\left(T_{n}\right)\right|} \frac{|h(T)|}{|T|} \leq C_{1} C_{2} B^{2} .
$$

Putting this into (13), we get for all $x \in T$ and all $n \geq 0$

$$
d_{h(T)}\left(h_{n}(x), h(x)\right) \leq \frac{1}{2} B^{3} C_{1} C_{2}|T|\left(M_{T_{n}}-1\right),
$$

and since $M_{T_{n}} \rightarrow 1$ as $n \rightarrow \infty$, the claim follows.

Using the Distortion Lemma one final time, we see that there exists $C_{3}>0$ such that for all $x, y \in T$ and all $n \geq 0$

$$
\left|\log \frac{\left|D h_{n}(x)\right|}{\left|D h_{n}(y)\right|}\right| \leq C_{3}|x-y|^{\alpha} .
$$

This shows us at once that the sequence $\left(D h_{n}\right)_{n \geq 0}$ is: (a) uniformly bounded (by the mean value theorem), as well as (b) equicontinuous, because in fact it is uniformly $\alpha$-Hölder. Therefore by the Arzelá-Ascoli theorem, $\left(D h_{n}\right)_{n \geq 0}$ is precompact in the $C^{\alpha}$-topology. If $\left(D h_{n_{j}}\right)$ is a convergent subsequence and $\varphi$ is its $\alpha$-Hölder limit, then, since by the claim above $h_{n_{j}} \stackrel{C^{0}}{\longrightarrow} h$ in $T$, we deduce by a standard $3 \epsilon$-argument that $h$ is differentiable in $T$ and that $D h \equiv \varphi$ there. As $T$ was arbitrary, the conjugacy is indeed $C^{1+\alpha}$, and the theorem is proved.

\section{ADDENDUM}

At the cost of making the proof less self-contained, we can actually discard Steps 2 and 3 in the following way. We know from the start that $h$ is quasisymmetric, so a fortiori it is Hölder of some exponent $0<s<1$ (cf. [Ah]). In the notation used in the proof, this gives us $\delta_{\varepsilon} \leq$ (const.) $\varepsilon^{s}$, which confronted with the expression of $\omega_{\varepsilon}$ following (11) yields $\omega_{\varepsilon} \leq$ (const.) $\varepsilon^{\beta}$ for all sufficiently small $\varepsilon$ and some $0<\beta<\alpha$. By a theorem of Carleson (cf. [Ca, Lemma 5]), $h$ must then be a $C^{1+\beta}$-diffeomorphism, and we can go directly to Step 4 .

\section{ACKNOWLEDGEMENTS}

The author wishes to thank Dennis Sullivan and Charles Tresser for several useful conversations on these and related matters, as well as the referee for suggesting various improvements.

\section{REFERENCES}

[Ah] L. Ahlfors, Lectures on quasiconformal mappings, Van Nostrand, Princeton, NJ, 1966. MR 34:336

[Ca] L. Carleson, On mappings conformal at the boundary, J. Analyse Math. 19 (1967), 1-13. MR 35:6821

[Cu] G. Cui, Linear models of circle expanding maps, Academia Sinica, preprint (1994).

[GS1] F. Gardiner and D. Sullivan, Symmetric structures on a closed curve, Amer. J. of Math. 114 (1992), 683-736. CMP 92:16 
[GS2] _ Lacunary series as quadratic differentials in conformal dynamics, Contemporary Mathematics 169 (1994). CMP 94:17

[PS] A. Pinto and D. Sullivan, The circle and the solenoid (to appear).

[Sa] R. Sacksteder, The measures invariant under an expanding map, Lecture Notes in Math., vol. 392, Springer-Verlag, Berlin, 1972, pp. 179-194. MR 53:11024

[SS] M. Shub and D. Sullivan, Expanding endomorphisms of the circle revisited, Erg. Th. \& Dynam. Sys. 5 (1985), 285-289. MR 87g:58104

[Su1] D. Sullivan, Differentiable structures on fractal-like sets, determined by intrinsic scaling functions on dual Cantor sets, Proc. Sympos. Pure Math. 48 (1988), 15-23. MR 90k:58141

[Su2] - Bounds, quadratic differentials and renormalization conjectures, Mathematics into the Twenty-First Century, Amer. Math. Soc. Centennial Publication, vol.2, Amer. Math. Soc., Providence, RI, 1991. MR 93k:58194

[Sh] M. Shub, Endomorphisms of compact differentiable manifolds, Amer. J. Math. 91 (1969), 175-199. MR 39:2169

Instituto de Matemática e Estatística, Universidade de São Paulo, Caixa Postal 66281, 05389-970 SÃo PaUlo SP- Brasil

E-mail address: edson@ime.usp.br 